\author{
Ching-Erh Lin ${ }^{1,2}$ \\ Hsu-Tun Cheng ${ }^{1}$ \\ I-Ju Fang ${ }^{1}$ \\ Yu-Chih Liu ${ }^{1}$ \\ Chia-Ming Kuo ${ }^{1}$ \\ Wann-Yin Lin ${ }^{1}$ \\ Chen-Hsing Lin ${ }^{2}$ \\ ${ }^{1}$ Department of Chemistry, \\ National Taiwan University, \\ Taipei, Taiwan \\ ${ }^{2}$ Department of Applied Chemistry, \\ Fooyin University, \\ Ta-Liao, Kau-hsiung County, Taiwan
}

Received September 6, 2005

Revised March 22, 2006

Accepted March 22, 2006
Research Article

\section{Strategies for enantioseparations of catecholamines and structurally related compounds by capillary zone electrophoresis using sulfated $\beta$-cyclodextrins as chiral selectors}

Strategies for simultaneous enantioseparations of three catecholamines (DL-norepinephrine, DL-epinephrine, and DL-isoproterenol) and three structurally related compounds (DL-octopamine, DL-synephrine, and DL-norephedrine) by CZE using sulfated $\beta$-CDs as chiral selectors were investigated. Four different separation modes were attempted: (I) using randomly sulfate-substituted $\beta-C D$ (MI-S- $\beta-C D)$ at relatively low concentrations in a high-concentration phosphate buffer at low $\mathrm{pH}$ in the normal polarity mode, (II) using MI-S- $\beta$-CD at high concentrations at low $\mathrm{pH}$ in the reversed polarity mode, (III) using MI-S- $\beta-C D$ at moderately high concentrations in a phosphate buffer at neutral $\mathrm{pH}$ in the normal polarity mode, and (IV) using the single isomer heptakis(2,3-dihydroxy-6-O-sulfo)- $\beta$-CD (SI-S- $\beta$-CD) at low to moderately high concentrations in a high-concentration $\mathrm{BGE}$ at low $\mathrm{pH}$ in the normal polarity mode. Among them, enantioseparation of these cationic solutes was best achieved under the conditions of mode (II). In mode (II) and mode (III), temperature is an important factor affecting the enantioresolution of norepinephrine. In mode (I) and mode (IV), the use of a high-concentration BGE $(150-200 \mathrm{mM})$ is crucial for effective enantioseparation of these cationic solutes with sulfated $\beta$-CDs. Comparative studies of enantioseparations of these cationic solutes with MI-S- $\beta$-CD and SI-S- $\beta$-CD reveal that the sulfate substituents of MI-S- $\beta-C D$ located at the $C_{2}$ - position interact strongly with the diol moiety of catecholamines.

Keywords: Capillary zone electrophoresis / Catecholamines / Chiral separation / Enantioseparation / Sulfated $\beta$-cyclodextrins

DOI 10.1002/elps.200500658

\section{Introduction}

In CE, the use of CDs and their derivatives as chiral selectors is the most common strategy employed for the separation of chiral compounds [1-5]. Enantioseparation of charged compounds has often been achieved with the use of neutral or charged CDs [6-20], or with dual CD

Correspondence: Professor Ching-Erh Lin, Department of Chemistry, National Taiwan University, Taipei 10671, Taiwan

E-mail: celin@ntu.edu.tw

Fax: +886-2-23636359

Abbreviations: CM- $\boldsymbol{\beta}-\mathbf{C D}$, carboxymethoxy- $\beta-C D$; MI-S- $\boldsymbol{\beta}-\mathbf{C D}$, randomly sulfate-substituted $\beta$-CD; SI-S- $\beta$-CD, the single isomer heptakis(2, 3-dihydroxy-6-O-sulfo)- $\beta$-CD; HP- $\beta$-CD, hydroxypropyl- $\beta-C D$ systems consisting of a charged $C D$ and a neutral CD [21-29], or two oppositely charged CDs [30]. Among many charged CDs studied to date, sulfated CDs have demonstrated the widest application range [6-8]. As the electrophoresis migration of the enantiomers of analytes can be modified through the complexation with CDs, enantioseparation can be achieved, provided that there are differences in binding constants and/or differences in the electrophoretic mobility of the enantiomeric complexes [31, 32].

Catecholamines are a group of $\beta$-phenylethylamine derivatives with hydroxyl groups substituted on the 3- and 4positions of the aromatic ring and on the $\beta$ position of the ethylamino side chain. These compounds act as neurotransmitters in the central and peripheral sympathetic 
nervous system. The interaction of these adrenergic agonists with their receptors is stereoselective. For catecholamines, such as norepinephrine and epinephrine, the enantiomer with the $(R)$-configuration is typically several hundred-fold more potent in agonistic effects than the one with the (S)-configuration [33]. It is also known that catecholamines and structurally related compounds exhibit dramatically different activity and selectivity. As enantioseparation of catecholamines and structurally related compounds is of great importance for biomedical research and pharmaceutical industry, the development of analytical methods for the effective enantioseparation of these analytes is desirable.

Applications of CE technique to the separation and/or determination of catecholamines have previously been reported [34-56]. For enantioseparation of catecholamines in CD-modified CE, the use of different derivatives of CD such as heptakis(2,6-di-O-methyl)- $\beta$-CD (HDM- $\beta$ CD) $[35,47,54]$, dimethyl- $\beta-C D$ [50], methyl- $\beta-C D$ [50], hydroxypropyl- $\gamma-\mathrm{CD} \quad(\mathrm{HP}-\gamma-\mathrm{CD}) \quad[48,50]$, carboxymethoxy- $\beta-C D(C M-\beta-C D)[35,50]$, sulfonated- $\beta-C D$ [49], and sulfated $\beta-C D$ [36] have appeared in the literature. Recently, enantioseparation of three neurotransmitters, including norepinephrine, epinephrine, and isoproterenol, was studied by CE equipped with microfabricated interdigitated electrodes using HDM- $\beta-C D$ as the chiral selector at $\mathrm{pH} 2.5$ [54]. Complete enantioresolution of racemic epinephrine and isoproterenol was achieved with sulfonated $\beta-\mathrm{CD}$ as chiral selector at low $\mathrm{pH}$ [49]. The enantiomers of norepinephrine and epinephrine could be completely separated by $\mathrm{CE}$ with amperometric detection employing CM- $\beta-C D$ as the chiral selector at $\mathrm{pH} 6.7$ [48]. However, simultaneous enantioseparation of these two analytes was not achievable [48]. For further improvement, the use of CD combined with crown ether [48] or with dendrimer [55] was considered.

In another study, negatively charged sulfated $\beta$-CDs were used by Gahm and Stalcup [36] to separate catecholamines and their precursors and to exploit chiral recognition mechanisms. The advantage of the reversed polarity mode at low $\mathrm{pH}$ for the enantioresolution of catecholamines and their precursors has been demonstrated. The use of high concentration charged CDs could not only shorten the analysis time but also enable to enhance enantioresolution of both weakly and strongly interacting racemates at the same time. However, due to overlapping of the enantiomeric peaks of norepinephrine and epinephrine, simultaneous enantioseparation was not satisfactory.

Recently, three different approaches for simultaneous enantioseparation of amphetamine type stimulants by CE using highly sulfated $\gamma$-CD as a chiral selector has been attempted [57]. Over the past decade, the single isomer sulfated CDs have widely been used as chiral resolving agents [8-21, 30, 58-62]. Depending on the positions and the degree of substitution of the sulfate group of CDs, they may possess unique enantioselectivity for chiral compounds and thus can offer possibilities for an enhancement of enantioselectivity and resolution for some analytes in chiral CE.

In view of these, it is desirable to study chiral separation of catecholamines in further detail with the use of sulfated $\beta$-CDs as chiral selectors. In the present study, four different approaches to chiral separation of three catecholamines, together with three structurally related compounds, by CZE using randomly sulfate-substituted $\beta-C D$ (MI-S- $\beta$-CD) and the single isomer heptakis(2,3-dihydroxy-6-O-sulfo)- $\beta$-CD (SI-S- $\beta-C D$ ) as chiral selectors are attempted. Comparative studies of enantioseparation of these cationic solutes with MI-S- $\beta-C D$ and SI-S- $\beta-C D$ are made. In addition, chiral recognition mechanisms are discussed.

\section{Materials and methods}

\subsection{Apparatus}

All CE separations were performed on a Beckman P/ACE 5500 equipped with a UV detector for absorbance measurements at $214 \mathrm{~nm}$ (Beckman Coulter, Fullerton, CA, USA). Uncoated fused-silica capillaries purchased from Polymicro Technologies (Phoenix, AZ, USA) were used. The dimensions of the capillary were $57 \mathrm{~cm} \times 50 \mu \mathrm{m}$ id. The effective length of the capillary was $50 \mathrm{~cm}$ from the injection end of the capillary. The CE system was interfaced with a microcomputer. System Gold software of Beckman was used for data acquisition. For $\mathrm{pH}$ measurements, a pH meter (Suntex Model SP-701, Taipei, Taiwan) was employed with a precision of $\pm 0.01 \mathrm{pH}$ unit.

\subsection{Chemicals and reagents}

Epinephrine, norepinephrine, synephrine, octopamine, and isoproterenol were obtained from Sigma (St. Louis, MO, USA). Norephedrine hydrochloride and MI-S- $\beta-C D$ were from Sigma-Aldrich (St. Louis, MO, USA). SI-S- $\beta-C D$ was purchased from ANTEK Instruments (Houston, TX, USA). All other chemicals were of analytical grade. Deionized water was prepared with a Milli-Q system (Millipore, Bedford, MA, USA).

Standard solutions of the analytes, with the exception of norephedrine, at a concentration of $20 \mu \mathrm{g} / \mathrm{mL}$ and those of norephedrine at concentrations of $50-150 \mu \mathrm{g} / \mathrm{mL}$ were 
prepared by dissolving analytes in an aqueous solution. The $\mathrm{pH}$ of a phosphate buffer was adjusted to the desired $\mathrm{pH}$ value by mixing various proportions of a certain concentration of sodium dihydrogenphosphate solution with the same concentration of phosphoric acid. All buffer solutions, freshly prepared weekly and stored in a refrigerator before use, were filtered through a membrane filter $(0.22 \mu \mathrm{m})$.

\subsection{Electrophoretic procedure}

When a new capillary was used, the capillary was washed for $30 \mathrm{~min}$ with $1.0 \mathrm{M} \mathrm{NaOH}$ solution, followed by a $30 \mathrm{~min}$ wash with deionized water at $25^{\circ} \mathrm{C}$. Before each injection, the capillary was prewashed for 3 min with running buffer and postwashed for $3 \mathrm{~min}$ with deionized water, for $3 \mathrm{~min}$ with $0.1 \mathrm{M} \mathrm{NaOH}$, and for $5 \mathrm{~min}$ with deionized water to maintain proper reproducibility of run-to-run injections. Sample injections were done in a hydrodynamic mode over $5 \mathrm{~s}$ under a pressure of $1.0 \mathrm{psi}$ at $25^{\circ} \mathrm{C}$. The measurements were run at least in triplicate to ensure reproducibility. An applied voltage of either $+20 \mathrm{kV}$ (in the normal polarity mode) or $-20 \mathrm{kV}$ (in the reversed polarity mode) for the phosphate buffer was selected to keep the total current at less than $100 \mu \mathrm{A}$. The detection wavelength was set either at $214 \mathrm{~nm}(\mathrm{pH} \mathrm{7.0)}$ or at $200 \mathrm{~nm}$ (pH 3.0.) Peak identification was conducted by spiking with the analyte to be identified. Mesityl oxide was used as the neutral marker.

\section{Results and discussion}

The structures of three catecholamines and three structurally related compounds, together with their $\mathrm{p} K_{\mathrm{a}}$ values, are given in Table 1. As shown in Fig. 1, four different separation modes were attempted for the enantioseparations of the six cationic solutes selected. Mode (I) uses MI-S- $\beta-C D$ as chiral selectors at relatively low concentrations with a high concentration phosphate buffer (150-200 mM) at low $\mathrm{pH}$ in normal polarity of the electrode. As the $\mathrm{p} K_{\mathrm{a}}$ values for the amino group of the test solutes are above 8.2, the analytes are positively charged at acidic $\mathrm{pH}$ owing to the protonation of the basic amino group. Thus free cationic solutes migrate toward the cathode. Under the conditions of mode (I), the effective electrophoretic mobility of the cationic solutes, even complexed with MI-S- $\beta-C D$ at low concentrations, remains to be positive. Mode (II) uses MI-S- $\beta$-CD at moderately high concentrations with a phosphate buffer at low $\mathrm{pH}$ in reversed polarity. Compared with the electrophoretic system in mode (I), the migration of the electrophoretic system is reversed. Mode (III) uses MI-S- $\beta-C D$
Table 1. The $p K_{a}$ values and structures of catecholamines and structurally related compounds studied

\begin{tabular}{|c|c|c|c|c|c|c|}
\hline & $\mathrm{p} K_{\mathrm{a}}^{\mathrm{a})}$ & $R_{1}$ & $R_{2}$ & $R_{3}$ & $R_{4}$ & $R_{5}$ \\
\hline Norepinephrine (1) & $\begin{array}{r}8.6 \\
9.8 \\
12.0\end{array}$ & $\mathrm{OH}$ & $\mathrm{OH}$ & $\mathrm{OH}$ & $\mathrm{H}$ & $\mathrm{H}$ \\
\hline Epinephrine (2) & $\begin{array}{c}8.58 \\
8.78 \\
10.0\end{array}$ & $\mathrm{OH}$ & $\mathrm{OH}$ & $\mathrm{OH}$ & $\mathrm{H}$ & $\mathrm{CH}_{3}$ \\
\hline Isoproterenol (3) & $\begin{array}{r}8.6 \\
10.1 \\
12.0\end{array}$ & $\mathrm{OH}$ & $\mathrm{OH}$ & $\mathrm{OH}$ & $\mathrm{H}$ & $\begin{array}{l}\mathrm{CH} \\
\left(\mathrm{CH}_{3}\right)_{2}\end{array}$ \\
\hline Octopamine (4) & $\begin{array}{l}8.88 \\
9.53\end{array}$ & $\mathrm{OH}$ & $\mathrm{H}$ & $\mathrm{OH}$ & $\mathrm{H}$ & $\mathrm{H}$ \\
\hline Synephrine (5) & $\begin{array}{r}9.3 \\
10.2\end{array}$ & $\mathrm{OH}$ & $\mathrm{H}$ & $\mathrm{OH}$ & $\mathrm{H}$ & $\mathrm{CH}_{3}$ \\
\hline Norephedrine (6) & & $\mathrm{H}$ & $\mathrm{H}$ & $\mathrm{OH}$ & $\mathrm{CH}_{3}$ & $\mathrm{H}$ \\
\hline
\end{tabular}

a) [66].

at moderately high concentrations with a phosphate buffer at neutral $\mathrm{pH}$ in normal polarity. As the effective electrophoretic mobility of the cationic solutes is smaller than the electroosmotic mobility of the BGE at neutral $\mathrm{pH}$, the apparent electrophoretic mobility of cationic solutes migrates toward the cathode. Mode (IV) uses SI-S- $\beta$-CD as a chiral selector at low to moderately high concentrations with a high concentration phosphate buffer (150-200 mM) at low $\mathrm{pH}$ in normal polarity. The effective electrophoretic mobility of cationic solutes with SI-S- $\beta$-CD is much smaller than that of the complexes with MI-S- $\beta-C D$. Apparently, the interaction of cationic solutes with SI-S- $\beta$-CD is comparatively much weaker than that with $\mathrm{MI}-\mathrm{S}-\beta-\mathrm{CD}$, thus, resulting in the positive effective electrophoretic mobility of cationic solutes as in the case of mode (I).

\subsection{Mode (I): MI-S- $\beta-C D$ at relatively low concentrations with a high-concentration phosphate buffer at low pH in the normal polarity mode}

As MI-S- $\beta-C D$ is composed of a number of MI-S- $\beta-C D$ (typically with substitution $7-11 \mathrm{~mol} / \mathrm{mol} \beta-\mathrm{CD}$ ), the concentration of MI-S- $\beta-C D$ is given in $\% \mathrm{w} / \mathrm{v}$, instead of $\mathrm{mM}$. 
Mode (I) : low MI-S- $\beta-C D$ concentration at low $\mathrm{pH}$

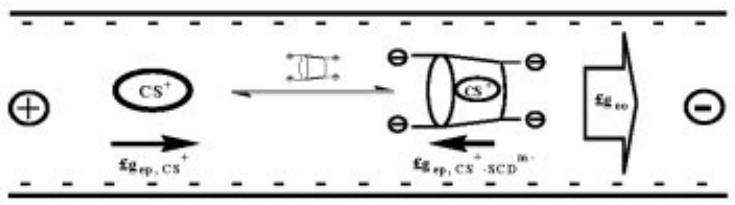

Mode (II) : high MI-S- $\beta-C D$ concentration at low $\mathrm{pH}$

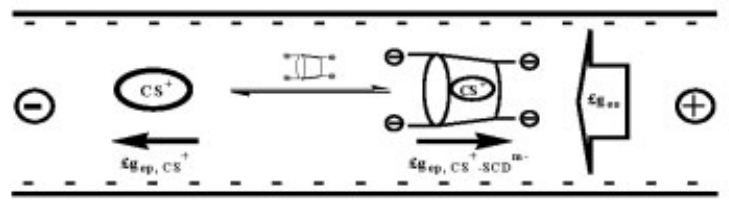

Mode (III) : high MI-S- $\beta-C D$ concentration at neutral pH

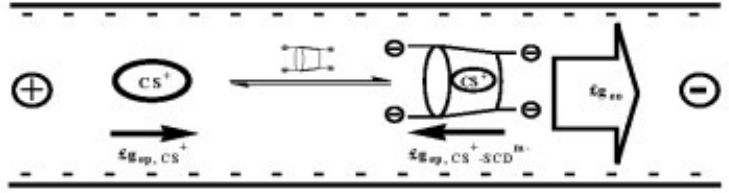

Mode (IV) : high SI-S- $\beta-C D$ concentration at low pH

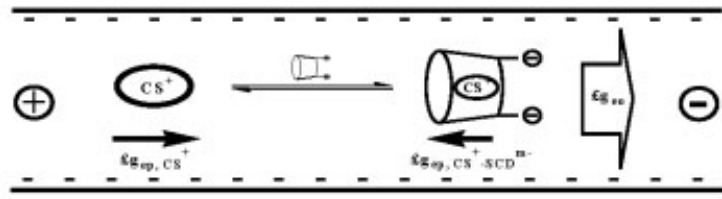

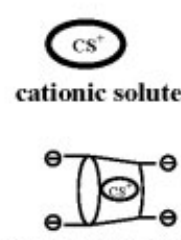

solute- MI- S- $\beta$-CD complexes
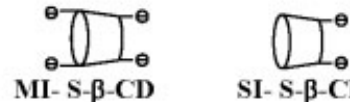

SI- S- $\beta-$ CD

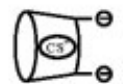

solute- SI- S- $\beta$-CD complexes
Figure 1. Schematic representation of four different separation modes: (I) low MI-S- $\beta-C D$ concentration with a high-concentration $\mathrm{BGE}$; at low $\mathrm{pH}$ in the normal polarity mode; (II) high MI-S- $\beta-C D$ concentration at low $\mathrm{pH}$ in the reversed polarity mode; (III) high $\mathrm{MI}-\mathrm{S}-\beta-\mathrm{CD}$ concentration at neutral $\mathrm{pH}$ in the normal polarity mode; (IV) low to moderately high SI-S- $\beta$-CD concentration with a highconcentration $\mathrm{BGE}$ at low $\mathrm{pH}$ in the normal polarity mode.

Figure 2 shows the electropherograms of cationic solutes obtained under mode (I) using a phosphate buffer (200 mM) containing MI-S- $\beta$-CD at relatively low concentrations at $\mathrm{pH}$ 3.0. For comparison, the electropherogram of these cationic solutes obtained using a phosphate buffer without the addition of chiral selector is also included (Fig. 2A). It was noted that enantioseparation of these cationic solutes was greatly affected by the

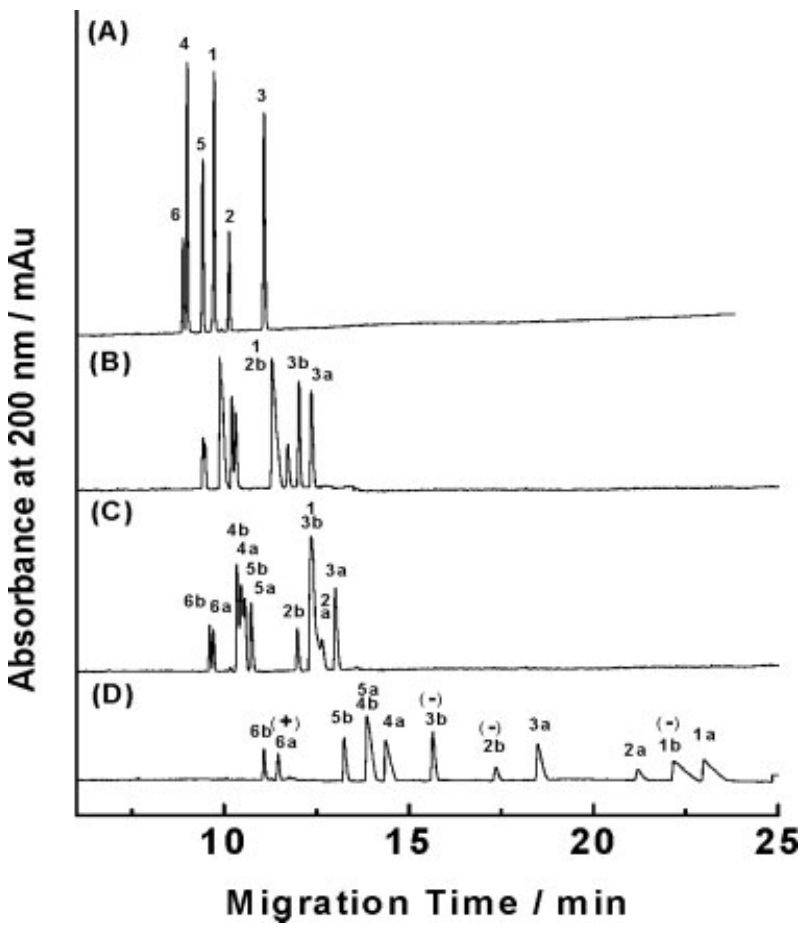

Figure 2. Electropherograms of six cationic solutes obtained under the conditions of mode (I) using a highconcentration phosphate buffer (200 mM) containing MIS- $\beta-C D$ at varied concentrations at $\mathrm{pH} 3.0$ in the normal polarity $(20 \mathrm{kV})$ : (A) $0 \%$, (B) $0.03 \%$, (C) $0.05 \%$, (D) $0.15 \%$ $\mathrm{w} / \mathrm{v}$. Detection wavelength, $200 \mathrm{~nm}$. Capillary, $57 \mathrm{~cm} \times 50 \mu \mathrm{m}$, id; sample concentration, $20 \mu \mathrm{g} / \mathrm{mL}$; other operating conditions, $20 \mathrm{kV}, 25^{\circ} \mathrm{C}$. Peak identification: 1 , norepinephrine; 2 , epinephrine; 3 , isoproterenol; 4 , octopamine; 5 , synephrine; 6 , norephedrine.

addition of MI-S- $\beta-C D$. This is especially true for epinephrine (2) and isoproterenol (3). With the addition of $0.03 \% \mathrm{w} / \mathrm{v}$ MI-S- $\beta-C D$, the enantiomers of epinephrine (2) and isoproterenol (3) were well resolved and the enantiomers of synephrine (5) were almost baseline-separated (Fig. 2B). Complete separation of the enantiomers of synephrine (5) and norephedrine (6) could be achieved with the addition of MI-S- $\beta-C D$ at $0.05 \%$ w/v (Fig. 2 C). By further increasing MI-S- $\beta-C D$ concentration to $0.09 \% \mathrm{w} / \mathrm{v}$, the enantiomers of octopamine (4) and synephrine (5) were completely separated and the enantiomers of norepinephrine (1) were almost baseline-separated (not shown). Reversal of the migration order of octopamine (4) and synephrine (5) was expected when the concentration of MI-S- $\beta-C D$ increased further. This is because octopamine (4), which possesses a primary amino group, interacts more strongly with MI-S- $\beta-C D$ than synephrine (5) which possesses a secondary amino group. In fact, with the addition of MI-S- $\beta-C D$ at $0.15 \% \mathrm{w} / \mathrm{v}$, the enantiomers of all of the six cationic solutes were well resolved (Fig. 2D). The migration of these cationic solutes toward the cath- 
ode follows the order norephedrine (6) <synephrine (5) <octopamine (4) <isoproterenol (3) <epinephrine (2) $<$ norepinephrine (1), with the "b" enantiomers migrating faster than the corresponding "a" enantiomers (Fig. 2D). However, the second enantiomer of synephrine (5a) comigrated with the first enantiomer of octopamine (4b); the first enantiomer of epinephrine (2b) migrated before the second enantiomer of isoproterenol (3a).

The enantiomeric peak of norephedrine designated as "6a" was spiked with the (+)-(S)-norephedrine and was experimentally confirmed. Similarly, the enantiomeric peaks designated as $1 \mathrm{~b}, 2 \mathrm{~b}$, and $3 \mathrm{~b}$ were spiked with (-)$(R)$-norepinephrine, $(-)-(R)$-epinephrine, and $(-)-(R)$-isoproterenol, respectively, and were experimentally confirmed. Accordingly, the enantiomeric peaks designated as "b" were assigned to the $(-)-(R)$-enantiomers, which migrated faster toward the cathode (with a greater positive effective electrophoretic mobility) than the corresponding $(+)-(S)$-enantiomers. Thus, the $(+)-(S)$-enantiomers of these cationic solutes bind more strongly with MI-S- $\beta-C D$.

It should be emphasized that the use of a BGE at a high concentration (150-200 mM) at low pH is crucial for effective enantioseparation of these cationic solutes in mode (I). It is noted that the optimal concentration of the phosphate buffer is in the range of 150-200 mM. By decreasing the concentration of the phosphate buffer from 200 to $150 \mathrm{mM}$, the separation was somewhat improved, but the migration time was prolonged to about $30 \mathrm{~min}$. By further lowering the concentration of phosphate buffer from 150 to $135 \mathrm{mM}$, the migration time was prolonged to about $50 \mathrm{~min}$. This is particularly true for the three catecholamines studied. In the presence of MI-S- $\beta-C D$, the migration times of the enantiomers of three catecholamines increase markedly and the enantioresolution of catecholamines enhances remarkably. This is understandable because the enantioresolution is directly proportional to the difference in the effective electrophoretic mobility of the enantiomers of an analyte and is inversely proportional to the square root of the sum of the electroosmotic mobility and the average of the effective electrophoretic mobility of the enantiomers [63]. On the contrary, it should be noted that, in the absence of MI-S- $\beta$ $C D$, the migration behavior of these cationic solutes is little affected by varying the concentration of phosphate buffer from 200 to $100 \mathrm{mM}$.

\subsection{Mode (II): MI-S- $\beta$-CD at moderately high concentrations at low $\mathrm{pH}$ in the reversed polarity mode}

A number of applications with the use of anionic CDs as chiral selectors in the reversed polarity mode under the condition of suppressed EOF have been reported in the literature $[9,10,25,36,64,65]$. Comparative studies on the peak shape and the resolution in the reversed polarity mode versus the normal polarity mode have found that preferred enantioseparations have been achieved for certain solutes by using the reversed mode [59, 64, 65]. Figure 3 shows the electropherograms of cationic solutes obtained with $\mathrm{MI}-\mathrm{S}-\beta-\mathrm{CD}$ at moderately high concentrations at $\mathrm{pH} 3.0$ in the reversed polarity mode at 25 and $40^{\circ} \mathrm{C}$. The enantiomer migration reversal was observed in comparison with the electropherogram obtained under the conditions of mode (I). This is consistent with the results obtained previously [36]. Sharp peaks were observed for the enantiomers of norepinephrine (1) which appeared first in the electropherogram. At $25^{\circ} \mathrm{C}$, with the addition of $2.0 \% \mathrm{w} / \mathrm{v}$ MI-S- $\beta$-CD in a phosphate buffer $(50 \mathrm{mM})$ at $\mathrm{pH} 3.0$, the enantiomeric peaks of norepinephrine (1) were partially resolved and the first enantiomeric peak of isoproterenol ( $3 a$ ) nearly comigrated with the first enantiomeric peak of octopamine (4a). As observed previously [36], complete separation of the enantiomers of norepinephrine (1) was not achievable by varying the concentration of $\mathrm{MI}-\mathrm{S}-\beta-\mathrm{CD}$ at $25^{\circ} \mathrm{C}$.

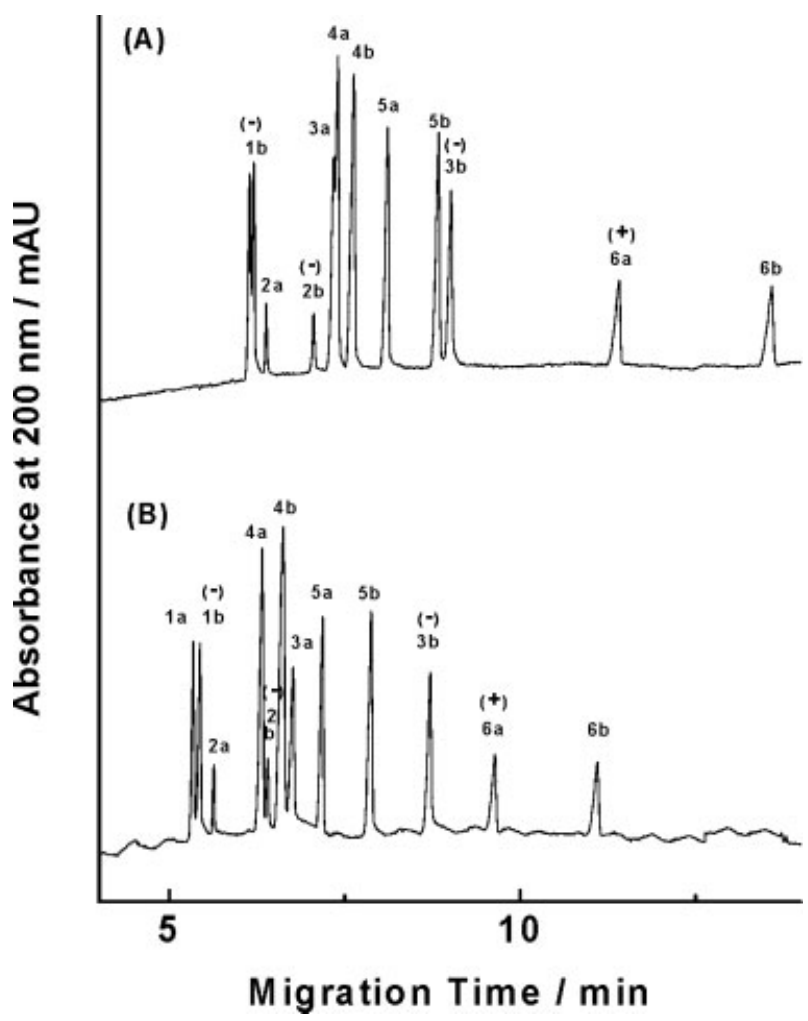

Figure 3. Electropherograms of six cationic solutes obtained under the conditions of mode (II) using a phosphate buffer $(50 \mathrm{mM})$ containing a high MI-S- $\beta-C D$ concentration $(2.0 \% \mathrm{w} / \mathrm{v})$ at $\mathrm{pH} 3.0$ in the reversed polarity mode $(-20 \mathrm{kV})$ at $(\mathrm{A}) 25^{\circ} \mathrm{C}$, and $(\mathrm{B}) 40^{\circ} \mathrm{C}$. Other operating conditions and peak identification are the same as for Fig. 2. 
It is worthy to point out that temperature is also an important factor for the optimization of enantioseparation of catecholamines in the reversed polarity mode. Moreover, with MI-S- $\beta-C D$ at higher concentrations at $40^{\circ} \mathrm{C}$, the analysis time was greatly shortened and the peak shape was significantly improved. As shown in Fig. 3B, by raising the operating temperature from 25 to $40^{\circ} \mathrm{C}$, baseline resolution could be nearly obtained for the enantiomers of norepinephrine (1) and complete enantioseparation of six cationic solutes could be simultaneously achieved within $13 \mathrm{~min}$. As illustrated, norephedrine (6), synephrine (5), and octopamine (4), which interact less strongly than catecholamines with MI-S- $\beta-C D$ under the conditions of mode (II) in this study, exhibit a much better enantioseparation in the reversed polarity mode.

\subsection{Mode (III): MI-S- $\beta$-CD at moderately high concentrations at neutral $\mathrm{pH}$ in the normal polarity mode}

Figure 4 shows the electropherogram of six cationic solutes obtained using MI-S- $\beta-C D$ at a moderately high concentration $(2.5 \% \mathrm{w} / \mathrm{v})$ at $\mathrm{pH} 7.0$ in the normal polarity mode. As expected, the effective electrophoretic mobility of cationic solutes decreases with increasing MI-S- $\beta-C D$ concentration. With the addition of MI-S- $\beta-C D$ at a concentration greater than $2.5 \% \mathrm{w} / \mathrm{v}$, the enantiomers of norepinephrine (1) were baseline-resolved and the enantiomers of the six cationic solutes studied could be simultaneously separated. Partial overlapping between the second enantiomeric peak of octopamine (4a) and the second enantiomeric peak of isoproterenol (3a) was observed. Severe to moderate peak broadening was observed for the enantiomers of norepinephrine (1) detected at $25^{\circ} \mathrm{C}$. However, the enantiomeric peaks could be substantially sharpened when the electrophoretic system was operated at $40^{\circ} \mathrm{C}$ (not shown).

It was found that, with MI-S- $\beta-C D$ at $2.5 \% \mathrm{w} / \mathrm{v}$, the migration follows the order norephedrine (6) < synephrine (5) <octopamine (4) for solutes without a diol structure and the order isoproterenol (3) <epinephrine (2) <norepinephrine (1) for catecholamines. As the extent of the variation of the mobility (or the migration time) of a cationic solute may reflect the interaction between the cationic solute and the chiral selector, the results indicate that catecholamines interact more strongly with MI-S- $\beta-C D$ than the other three cationic solutes without the diol moiety. Among them, norepinephrine (1) which possesses a primary amino group binds most strongly with MI-S- $\beta$ $\mathrm{CD}$. The alkylation of the amino group resulted in the reduction of interaction between catecholamines and MI$S-\beta-C D$. Bulky isopropyl substitution on the amino group further reduces the binding strength of isoproterenol (3) to

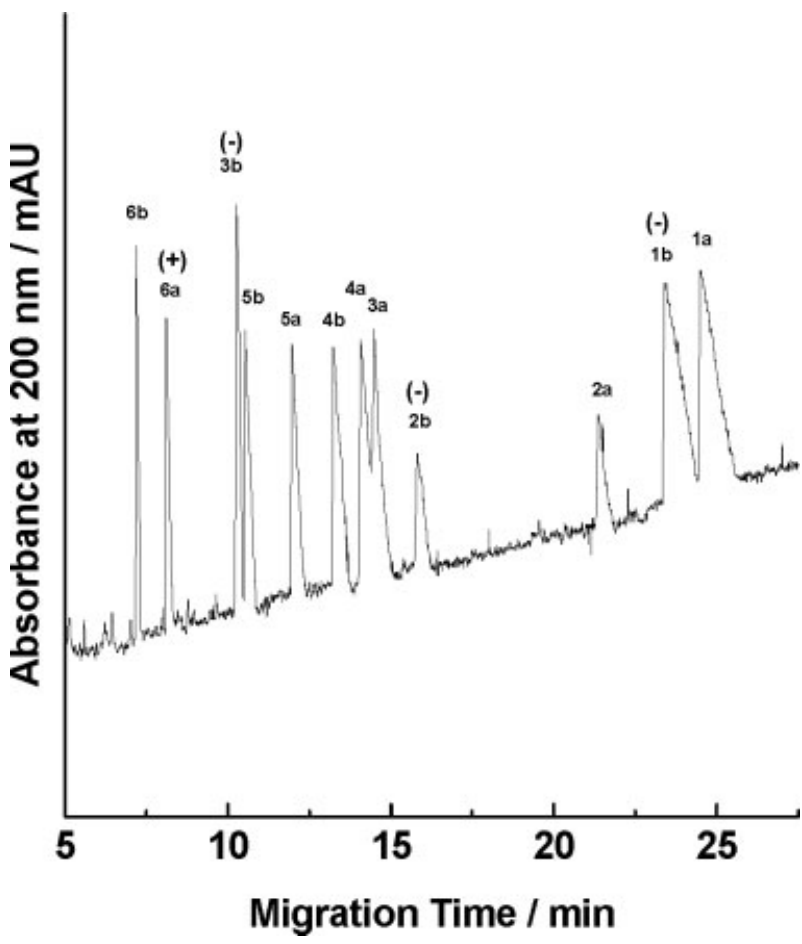

Figure 4. Electropherogram of cationic solutes obtained under the conditions of mode (III) by using a phosphate buffer $(50 \mathrm{mM})$ containing $2.5 \% \mathrm{w} / \mathrm{v}$ MI-S- $\beta$-CD at $\mathrm{pH} 7.0$ in the normal polarity mode. Detection wavelength $214 \mathrm{~nm}$; other operating conditions and peak identification are the same as for Fig. 2.

MI-S- $\beta$-CD. The positive contribution of the hydroxyl group substituted on the 3-position of the phenyl ring to the binding strength of catecholamines with MI-S- $\beta-C D$ was supported by comparison of the results obtained for norepinephrine (1) with octopamine (4) and for epinephrine (2) with synephreine (5). In contrast, the lack of hydroxyl substitutions at the 3- and 4-positions of the phenyl ring resulted in the lowest interaction of norephedrine (6) with MI-S- $\beta$-CD among the six cationic solutes studied.

It is worthy to note that the enantioresolution of epinephrine (2) is much greater than that of norepinephrine (1), but the enantioresolution of isoproterenol (1) is smaller than that of epinephrine (2). The results reveal that the alkylation of the amino group enhances the enantioresolution of catecholamines with MI-S- $\beta-C D$, but a bulky isopropyl substituent in the tertiary amino group is not as favorable as the secondary amino group in chiral discrimination. Apparently, the steric effect between isoproterenol (3) and MI-S- $\beta-C D$ on the enantioresolution is indicative. The results also reveal that a greater interaction between the chiral selectand and chiral selector does not necessarily mean to have a better chiral discrimination. 


\subsection{Mode (IV): SI-S- $\beta$-CD at low to moderately high concentrations in a high concentration BGE at low pH in the normal polarity mode}

Figure 5 shows the electropherograms of cationic solutes obtained under the conditions of mode (IV) using phosphate buffer at a high concentration (150-200 mM) containing SI-S- $\beta$-CD at low to moderately high concentrations at pH 3.0 in the normal polarity mode. Baseline resolution could be achieved with the addition of $0.4 \% \mathrm{w} / \mathrm{v}$ SI-S- $\beta$-CD for isoproterenol (3), while the enantiomers of epinephrine (2) are only partially resolved. Chiral discrimination was most effective for the enantiomers of isoproterenol (3) with SI-S- $\beta$-CD, instead of epinephrine (2). Compared with the results obtained with MI-S- $\beta-C D$, the lack of sulfate substitutions at the $\mathrm{C}_{2}-$ and/or $\mathrm{C}_{3^{-}}$ positions on the wider rim of SI-S- $\beta$-CD enhances the enantioresolution of isoproterenol (3) (Fig. 5A). As the addition of SI-S- $\beta$-CD at a relatively much higher concentration is required for an effective enantioseparation of isoproterenol (3), the interaction of isoproterenol (3) with SI-S- $\beta$-CD is much weaker than that with MI-S- $\beta-C D$. Effective enantioseparation of epinephrine (2) was achieved with the addition of $0.7 \% \mathrm{w} / \mathrm{v}$ SI-S- $\beta$-CD (not shown), whereas the enantiomers of synephrine (5) were baseline resolved with addition of SI-S- $\beta$-CD at $0.8 \% \mathrm{w} / \mathrm{v}$ (Fig. 5B). By further increasing SI-S- $\beta$-CD concentration to $1.0 \% \mathrm{w} / \mathrm{v}$, the enantiomers of octopamine (4) and norepinephrine (1) were almost baseline resolved (Fig. 5C). Unfortunately, no enantioseparation of norephedrine (6) could be achieved with the addition of SI-S- $\beta$-CD up to $1.3 \% \mathrm{w} / \mathrm{v}$.

The variations of the electrophoretic mobility of the six cationic solutes studied as a function of SI-S- $\beta$-CD concentration in the range of $0-1.3 \% \mathrm{w} / \mathrm{v}$ using $200 \mathrm{mM}$ phosphate buffer at pH 3.0 were studied (not shown). As the extent of the variation of the mobility of cationic solutes may reflect the interactions between the cationic solutes and chiral selector, the results of two distinct sets of mobility curves indicate that SI-S- $\beta$-CD interacts much less strongly with catecholamines, which have a less extent of the variations of the electrophoretic mobility, than with octopamine (4), synephrine (5), and norephedrine (6). The binding strength of catecholamines to SI-S$\beta-C D$ is considerably smaller than that of catecholamines to $\mathrm{MI}-\mathrm{S}-\beta-\mathrm{CD}$. As MI-S- $\beta-\mathrm{CD}$ possesses sulfate-substituents at the $\mathrm{C}_{2}$-position (and perhaps $\mathrm{C}_{3}$-position) of $\beta-C D$ [66], whereas SI-S- $\beta$-CD does not, the results indicate that the diol moiety of catecholamines may interact strongly with the sulfate-substituents of MI-S- $\beta-C D$ located at the $\mathrm{C}_{2}$-position (and perhaps $\mathrm{C}_{3}$-position). Compared with the results obtained with MI-S- $\beta-C D$, the weakening of the binding of catecholamines with

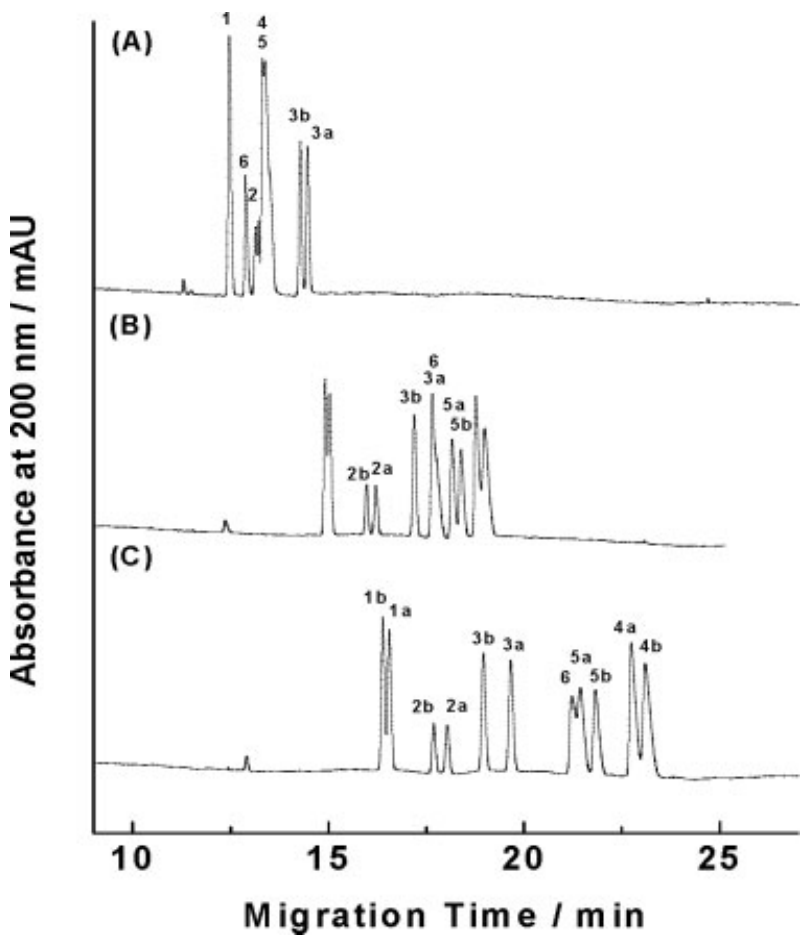

Figure 5. Electropherograms of six cationic solutes obtained under the conditions of mode (IV) using a highconcentration phosphate buffer $(200 \mathrm{mM})$ containing SIS- $\beta$-CD at varied concentration at $\mathrm{pH} 3.0$ in the normal polarity mode: (A) $0.4 \%$, (B) $0.8 \%$, and (C) $1.0 \% \mathrm{w} / \mathrm{v}$. Other operating conditions and peak identification are the same as for Fig. 2.

SI-S- $\beta$-CD is probably partly due to the steric effect of the diol moiety of catecholamines and/or partly due to an increased instability of catecholamines in the cavity of SIS- $\beta-C D$.

As in the case of mode (I), the use of a phosphate buffer at high concentrations (150-200 mM) and at low $\mathrm{pH}$ is crucial for effective enantioseparation of these cationic solutes within an acceptable analysis time. It is noted that the migration behavior of cationic solutes without the diol moiety was significantly affected by the concentration of phosphate buffer. By decreasing the concentration of phosphate buffer from 200 to $120 \mathrm{mM}$, norephedrine (6) and synephrine (5) could be baseline-separated, but the analysis time increased markedly from 27 to about 50 min (not shown). The enantiomeric peaks of octapamine (4) could not be detected in 60 min with the use of a phosphate buffer at $120 \mathrm{mM}$. As the interactions of cationic solutes with SI-S- $\beta$-CD in mode (IV) are about ten times smaller than those with MI-S- $\beta-C D$, the results clearly demonstrate that the sulfate substituents located at the $\mathrm{C}_{2}$ (and/or $\mathrm{C}_{3}$ ) position of MI-S- $\beta-\mathrm{CD}$ play an important role in enantioseparation and chiral recognition of these catecholamines. 


\section{Concluding remarks}

The enantiomers of six selected cationic solutes were effectively separated using sulfated $\beta-C D$ s as chiral selectors. Among four different approaches attempted, enantioseparation of these cationic solutes was best achieved in the reversed polarity mode at low $\mathrm{pH}$. Temperature is an important factor affecting the enantioresolution of norepinephrine. For effective enantioseparation of these six cationic solutes with sulfated $\beta$-CDs, the use of a BGE at high concentrations and at low $\mathrm{pH}$ in the normal polarity is crucial. Comparative studies of enantioseparations of these cationic solutes with MI-S- $\beta$ CD and SI-S- $\beta$-CD reveal that sulfate substituents of MIS- $\beta$-CD located at the $\mathrm{C}_{2}$-position (and perhaps $\mathrm{C}_{3}$-position), which interact strongly with the diol moiety of catecholamines, may play an important role in enantioseparation and chiral recognition of catecholamines.

We thank the National Science Council of Taiwan for financial support.

\section{References}

[1] Khaledi, M. G. (Ed.), High Performance Capillary Electrophoresis, Wiley, New York, NY 1998.

[2] Landers, J. (Ed.), Handbook of Capillary Electrophoresis, CRC Press, Boca Raton, FL 1997.

[3] Chankvetadze, B. (Ed.), Capillary Electrophoresis in Chiral Analysis, Wiley, Chichester, UK 1997.

[4] Fanali, S., J. Chromatogr. A 2000, 875, 89-122.

[5] Vigh, Gy., Sokolowski, A. D., Electrophoresis 1997, 18, 2305-2310.

[6] Evans, C. E., Staleup, A. M., Chirality 2003, 15, 709-723.

[7] de Boer, T., de Zeeuw, R. A., de Jong, G. J., Ensing, K., Electrophoresis 2000, 21, 3220-3239.

[8] Fillet, M., Hubert, Ph., Crommen, J., J. Chromatogr. A 2000, $875,123-134$.

[9] Staleup, A. M., Gahm, K. H., Anal. Chem. 1996, 68, 13601368.

[10] Gratz, S. R., Staleup, A. M., Anal. Chem. 1998, 70, 51665171.

[11] Wu, W., Staleup, A. M., J. Liq. Chromatogr. 1995, 18, 12891315.

[12] Tait, R. J., Thompson, D. O., Stella, V. J., Stobaugh, J. F., Anal. Chem. 1994, 66, 4013-4018.

[13] Nishi, H., Terabe, S., J. Chromatogr. A 1995, 694, 245-276.

[14] Chankvetadze, B., J. Chromatogr. A 1997, 792, 269-295.

[15] Lelievre, F., Gareil, P., Bahaddi, V., Galons, H., Anal. Chem. 1997, 69, 393-401.

[16] Izumoto, S. I., Nishi, H., Electrophoresis 1999, 20, 189-197.

[17] Surapaneni, S., Ruterbories, K., Lindstrom, T., J. Chromatogr. A 1997, 761, 249-257.

[18] Lin, S. L., Lin, C. E., J. Chromatogr. A 2004, 1032, 213-218.

[19] Lin, C. E., Lin, S. L., Liao, W. S., Liu, Y. C., J. Chromatogr. A 2004, 25, 2786-2795.

[20] Lin, C. E., Lin, S. L., Fang, I. J., Liao, W. S., Chen, C. C., Electrophoresis 2004, 25, 2786-2795.
[21] Abushoffa, A. M., Fillet, M., Hubert, P., Commen, J., J. Chromatogr. A 2002, 948, 321-329.

[22] Fillet, M., Chankvetadze, B., Crommen, J., Blaschke, G., Electrophoresis 1999, 20, 2691-2697.

[23] Fillet, M., Fotsing, L., Crommen, J., J. Chromatogr. A 1998, 817, 113-119.

[24] Lelievre, F., Gareil, P., Jardy, A., Anal. Chem. 1997, 69, 385392.

[25] Gahm, K. H., Chang, L. W., Armstrong, D. W., J. Chromatogr. A 1997, 759, 149-155.

[26] Lurie, I. S., J. Chromatogr. A 1997, 792, 297-307.

[27] Lurie, I. S., Klein, R. F., Dal Cason, T. A., LeBelle, M. J. et al.,Anal. Chem. 1994, 66, 4019-4026.

[28] Sepaniak, M. J., Copper, C. L., Whitaker, K. W., Anigbogu, V. C., Anal. Chem. 1995, 67, 2037-2041.

[29] Anigbogu, V. C., Copper, C. L., Sepaniak, M. J., J. Chromatogr. A 1995, 705, 343-349.

[30] Abushoffa, A. M., Fillet, M., Servais, A. C., Hubert, P., Crommen, J., Electrophoresis 2003, 24, 343-350.

[31] Biggin, M. E., Williams, R. L., Vigh, Gy., J. Chromatogr. A 1995, 692, 319-325.

[32] Williams, R. L., Vigh, Gy., J. Chromatogr. A 1995, 716, 197205.

[33] Delgado, J. N., Remers, W. A. (Eds.), Textbook of Organic Medicinal and Pharmaceutical Chemistry, 10 Edn., Lippincott-Raven, Philadelphia, 1998.

[34] Dette, C., Ebel, S., Terabe, S., Electrophoresis 1994, 15, 799-803.

[35] Hong, S., Lee, C. S., Electrophoresis 1995, 16, 2132-2136.

[36] Gahm, K. H., Stalcup, A. M., Chirality 1996, 8, 316-324.

[37] Fanali, S., Furlanetto, S., Aturki, Z., Pinzauti, S., Chromatographia 1998, 48, 395-401.

[38] Wang, F., Khaledi, M. G., J. Microcol. Sep. 1999, 11, 11-21.

[39] Zhong, M., Zhou, J., Lunte, S. M., Zhao, G. et al.,Anal. Chem. 1996, 68, 203-207.

[40] Zhu, R., Kok, W. T., Anal. Chem. 1997, 69, 4010-4016.

[41] Siren, H., Karjalainen, U., J. Chromatogr. A 1999, 853, 527533.

[42] Schmid, M. G., Laffranchini, M., Dreveny, D., Gubitz, G., Electrophoresis 1999, 20, 2458-2461.

[43] Britz-Mckibbin, P., Wong, J., Chen, D. D. Y., J. Chromatogr. A 1999, 853, 535-540.

[44] Park, Y. H., Zhang, X., Rubakhin, S. S., Sweedler, J. V., Anal. Chem. 1999, 71, 4997-5002.

[45] Kartsova, L. A, Sidorova, A. A., Kazakov, V. A., Bessonova, E. A, Yashin, A. Ya., J. Anal. Chem. 2004, 59, 737-741.

[46] Chicharro, M., Sanchez, A., Zapardiel, A., Rubianes, M. D., Rivas, G., Anal. Chim. Acta 2004, 523, 185-191.

[47] Wei, W., Luo, G., Yan, C., Sun, Z., J. High Resolut. Chromatogr. 1998, 21, 440-444.

[48] Schwarz, M. A., Hauser, P. C., J. Chromatogr. A 2001, 928, 225-232.

[49] Yang, W. C., Yu, A. M., Yu, X. D., Chen, H. Y., Electrophoresis 2001, 22, 2025-2031.

[50] Maruszaka, W., Trojanowicza, M., Margasinska, M., Engellhardt, H., J. Chromatogr. A 2001, 926, 327-336.

[51] Liu, Z., Niwa, O., Kurita, R., Horiuchi, T., Anal. Chem. 2000, $72,1315-1321$.

[52] Garcia, A., Heinaenen, M., Jimenez, L. M., Barbas, C., J. Chromatogr. A 2000, 871, 341-350.

[53] Klett, O., Nischang, I., Nyholm, L., Electrophoresis 2002, 23, 3678-3682. 
[54] Male, K. B., Luong, J. H. T., J. Chromatgr. A 2003, 1003, 167-178.

[55] Schwoorz, M. A., Hauser, P. C., Anal. Chem. 2003, 75, 46914695.

[56] Constantin, S., Bicker, W., Zarbl, E., Lammerhofer, M., Lindner, W., Electrophoresis 2003, 24, 1668-1679.

[57] Iwata, Y. T., Garcia, A., Kanamori, T., Inoue, H. et al.,Electrophoresis 2002, 23, 1328-1334.

[58] Vicent, J. B., Sokolowski, A. D., Nguyen, T. V., Vigh, Gy., Anal. Chem. 1997, 69, 4226-4233.

[59] Vicent, J. B., Kirby, D., Nguyen, T. V., Vigh, Gy., Anal. Chem. 1997, 69, 4419-4428.
[60] Cai, H., Nguyen, T. V., Vigh, Gy., Anal. Chem. 1998, 70, 580589.

[61] Zhu, W., Vigh, Gy., Electrophoresis 2001, 22, 1394-1398.

[62] Zhu, W., Vigh, Gy., Electrophoresis 2003, 24, 130-138.

[63] Jorgenson, J. W., Lukacs, K. D., Anal. Chem. 1981, 53, 1298-1302.

[64] Dolezalova, M., Fanali, S., Electrophoresis 2000, 21, 32643269.

[65] Chen, F. T., Shen, G., Evangelista, R. A., J. Chromatogr. A 2001, 924, 523-532.

[66] Drayton, C. J., Comprehensive Medicinal Chemistry Vol. 6, Pergamon Press, Oxford 1990. 